\title{
Gravitational-wave signature of an inspiral into a supermassive horizonless object
}

\author{
Michael Kesden, Jonathan Gair, and Marc Kamionkowski \\ California Institute of Technology, Mail Code 130-33, Pasadena, California 91125, USA
}

(Received 16 November 2004; published 10 February 2005)

\begin{abstract}
Event horizons are among the most intriguing of general relativity's predictions. Although on firm theoretical footing, direct indications of their existence have yet to be observed. With this motivation in mind, we explore here the possibility of finding a signature for event horizons in the gravitational waves (GWs) produced during the inspiral of stellar-mass compact objects (COs) into the supermassive ( $10^{6} M_{\odot}$ ) objects that lie at the center of most galaxies. Such inspirals will be a major source for LISA, the future space-based GW observatory. We contrast supermassive black holes with models in which the central object is a supermassive boson star (SMBS). Provided the COs interact only gravitationally with the SMBS, stable orbits exist not just outside the Schwarzschild radius but also inside the surface of the SMBS as well. The absence of an event horizon allows GWs from these orbits to be observed. Here we solve for the metric in the interior of a fairly generic class of SMBS and evolve the trajectory of an inspiraling CO from the Schwarzschild exterior through the plunge into the exotic SMBS interior. We calculate the approximate waveforms for GWs emitted during this inspiral. Geodesics within the SMBS surface will exhibit extreme pericenter precession and other features making the emitted GWs readily distinguishable from those emitted during an inspiral into a black hole.
\end{abstract}

DOI: 10.1103/PhysRevD.71.044015

PACS numbers: 04.30.Db, 04.25.Nx, 04.80.Nn, 95.35.+d

\section{INTRODUCTION}

The black-hole event horizon, a surface from beyond which no information can be received, is one of the most intriguing predictions of general relativity. From the theoretical point of view, the prediction is on a fairly firm footing. However, event horizons have become so central to physics and astrophysics that efforts to obtain direct empirical evidence for their existence are certainly warranted. In other words, is there any observation or measurement that we can make that would allow us to "see" the event horizon?

This question has motivated a small body of work in which the phenomenology of accretion onto alternatives to stellar-mass or supermassive black holes (SMBHs) has been worked out [1,2]. X-ray spectroscopy or $\mu$-arcsec imaging might distinguish the accretion disks of black holes from those surrounding more exotic alternatives [3]. A second approach involves the "shadow" cast on background sources by the black hole when acting as a strong gravitational lens [4]. Current observations are within a factor of 2 of being able to resolve the shadow of Sgr A* using very long baseline interferometry (VLBI) in the submillimeter. Although these calculations may not be applicable if the predictions of general relativity hold true on astrophysical scales, such work can be interesting in its own right and sometimes illuminates certain aspects of the phenomenology of the standard black-hole spacetime.

With these motivations in mind, we study here a third possibility, namely, whether gravitational-wave (GW) signals from the inspiral of stellar-mass compact objects (COs) into the supermassive objects at galactic centers may ultimately be used to ascertain the existence of event horizons. There are in fact good prospects of detecting
GWs from such extreme-mass-ratio inspirals (EMRIs). Measurements of stellar velocities within galactic cusps imply that the dynamics within a few parsecs of the center are dominated by a supermassive object $[5,6]$. Stellar clusters sufficiently dense to enclose enough mass within the observationally determined volume would have lifetimes much less than the age of the Galaxy [7] leading most to believe that the object must be compact; i.e., a SMBH. Still, stellar-dynamical measurements probe distances much larger than the Schwarzschild radius, and thus cannot distinguish SMBHs from sufficiently compact alternatives. Although future stellar-dynamical observations will probe smaller radii, they will never probe the spacetime structure anywhere even close to the horizon [8].

These supermassive compact objects are expected to capture stars from the surrounding galactic cusps $[9,10]$. Main-sequence stars will generally be disrupted at a tidal radius $r_{t} \approx 2\left(M / m_{*}\right)^{1 / 3} r_{*}$, where $m_{*}$ and $r_{*}$ are the stellar mass and radius and $M$ the mass of the supermassive central object. For stars with a solar mass and radius, this implies $r_{t} \approx 50 R_{s} M_{6}^{-2 / 3}$ with $M_{6}$ the mass of the central object in $10^{6} M_{\odot}$. Thus main-sequence stars are tidally disrupted long before they reach the Schwarzschild radius $R_{s}=2 G M / c^{2}$. However, an evolved stellar population will also contain a fraction of white dwarfs, neutron stars, and stellar-mass black holes that, owing to their smaller radii, can maintain their integrity down to the innermost stable orbit (ISO) and beyond. Under the extreme-massratio approximation, well justified for the case $\eta \equiv$ $m_{*} / M \lesssim 10^{-6}$, these COs will travel along geodesics of the central object. However, the galactic cusp is a very crowded environment, and two-body scattering will change the orbital parameters of the COs over a relaxation time $t_{r}$. Dynamical friction will cause heavy objects such 
as neutron stars and stellar-mass black holes to sink to the center of the cusp. Eventually, the COs will enter the critical region of phase space known as the loss cone in which the time scale for the loss of energy due to gravitational radiation is less than the time scale for two-body scattering out of the loss cone. After this point, the evolution of the orbit should be entirely determined by the loss of energy and angular momentum in GWs, ultimately leading to capture by the central object. The expected rate of such captures is highly model-dependent, subject to uncertainties in the galaxy luminosity function, the mass function of central objects, and the initial stellar-mass function (IMF) among other variables. Conservatively, event rates of order $10^{-8}$ per galaxy per year are anticipated, implying 0.1 captures per year out to $1 \mathrm{Gpc}$ [9]. This result could be enhanced by an order of magnitude by a top-heavy IMF, either due to low metallicity in the early universe or starbursts in the high-density environment of the galactic cusp itself [10]. EMRIs about a $10^{6} M_{\odot}$ central object would produce GWs in the frequency band $10^{-4}$ to $10^{-2} \mathrm{~Hz}$ probed by LISA, making them an interesting subject for theoretical investigation.

To perform this investigation, we need to contrast a Schwarzschild black hole with a specific candidate whose spacetime is identical to Schwarzschild at large distances but merges smoothly onto a horizonless solution in an interior region. For this purpose, we adopt as a "straw man" the spacetime of a supermassive boson star (SMBS) whose radius is only a few times the Schwarzschild radius. Such a star consists of a coherent scalar-field configuration supported against gravity by its own self-interaction. Although no fundamental scalar fields have yet been discovered, the fertile imaginations of particle theorists have provided no shortage of candidates; e.g., the standard-model Higgs field, the squark, slepton, and sneutrino fields in supersymmetric models, the axion field, and the dilaton in supergravity models. We restrict our attention to (nontopological) soliton stars, which are characterized by interaction potentials for which bound, stable solutions exist even in the absence of gravity [11]. This model allows us to choose parameters for the scalar-field Lagrangian so that a $\sim 10^{6} M_{\odot}$ SMBS emerges for massive parameters of order $100 \mathrm{GeV}$. This is not to suggest, however, that such a SMBS is necessarily likely to arise within the standard model or its most natural extensions. SMBSs with similar structure can just as easily arise with vastly different mass scales in other models, including "mini-boson stars" [12], nonspherical (but axially symmetric) scalar-field configurations partially supported against gravitational collapse by angular momentum [13], and (nonsolitonic) boson stars in which a massive scalar field is held up against gravitational collapse by a quartic self-interaction [14].

How might the EMRI into such a SMBS differ from that into a SMBH? The famous "no-hair" theorem states that the properties of an uncharged black hole are uniquely determined by the hole's mass $M$ and spin $a$. Any stationary, axisymmetric metric can be expanded in terms of mass and current multipole moments $M_{l}$ and $S_{l}$ [15]. For the Kerr metric, the "no-hair" theorem implies that all multipole moments can be expressed in terms of the mass and spin, $M_{l}+i S_{l}=M(i a)^{l}$. By contrast, no such strict relation need exist between the multipole moments of a generic boson-star metric. For example, Ryan [16] showed that all the multipole moments of the central object can be extracted from the gravitational waveform produced during an EMRI, even in the restricted case of circular orbits in the equatorial plane. As the orbital frequency $\Omega$ increases during inspiral, the number of radians of orbital motion per logarithmic frequency interval $d \Phi / d(\ln \Omega)$ can be calculated in a power series in $\Omega$. The coefficients of this power series are simple polynomial functions of the multipole moments. Ryan applied this formalism to spinning boson stars whose mass-quadrupole moment $\left|M_{2}\right|$ greatly exceeded $M a^{2}$, the value expected for a black hole of comparable mass and spin [13]. The spherical boson stars we consider here have perfectly Schwarzschild spacetimes outside of their surfaces, making them indistinguishable from black holes during the early stages of an EMRI. We rely instead on the GWs produced following the final plunge from the ISO into the central object itself. For a black hole, the presence of an event horizon precludes all observations of the inspiraling $\mathrm{CO}$ subsequent to the final plunge. After a brief "ringdown" period, the black hole ceases to be a significant source of gravitational radiation. For boson-star inspirals however, many orbits within the boson-star interior are expected provided that the $\mathrm{CO}$ interacts only gravitationally with the scalar field. For compact boson stars with surfaces interior to the ISO, circular orbits can develop an extremely large post-plunge eccentricity, leading to a sudden excitation of higher-order harmonics of the fundamental frequency $f \equiv \Omega / \pi$. As the $\mathrm{CO}$ spirals deeper into the boson-star potential well, we expect the fundamental frequency to decrease as less mass is enclosed by smaller orbits. By explicitly comparing the waveforms produced during black-hole and boson-star inspirals, we hope to determine how effectively they can be differentiated.

A final caveat to consider is whether the accumulation of a large mass at the center of the boson star either through inspirals or accretion will cause it to collapse into a black hole. While we expect such a collapse to occur beyond a certain mass limit, the calculation of this collapse is highly model-dependent and beyond the scope of this paper. Such collapses may affect the event rates of boson-star inspirals, but will not alter either the dynamics or waveforms which are the subject of this paper.

The paper is organized as follows. In Sec. II, we describe the particular boson-star model examined in this paper as originally formulated by Ref. [17]. Spherically symmetric 
solutions for the metric and scalar field are obtained from the Euler-Lagrange and Einstein equations. In Sec. III, we determine the geodesics of the boson-star metric, and consider qualitatively the possible allowed trajectories for the $\mathrm{CO}$ during inspiral. We then quantify this approach in Sec. IV by presenting a model for the loss of energy and angular momentum to gravitational radiation. The CO's orbital parameters are then evolved in light of these radiative losses in Sec. V. Calculated trajectories and waveforms for several initial conditions are displayed in Sec. VI, and some final remarks on the limitations of our approach and open questions that need to be addressed are given in Sec. VII. A brief appendix examines the accuracy of the quadrupole approximation near the ISO.

\section{BOSON-STAR MODEL}

Two requirements must be satisfied for a scalar field to have nontopological-soliton solutions [11]. The first of these requirements is the existence of an additive conservation law, which by Noether's theorem can be guaranteed by a symmetry of the Lagrangian. In the model of Friedberg, Lee, and Pang [17] adopted in this paper, the Lagrangian density $\mathfrak{L}$ is invariant under a global phase transformation $\phi \rightarrow e^{i \theta} \phi$ of the complex scalar field $\phi$,

$$
\mathfrak{L}=-\phi^{\dagger \mu} \phi_{\mu}-U\left(\phi^{\dagger} \phi\right),
$$

where a dagger denotes Hermitian conjugation and $\phi_{\mu} \equiv$ $\partial \phi / \partial x^{\mu}$. Such a Lagrangian will possess a conserved Noether current

$$
j^{\mu} \equiv-i\left(\phi^{\dagger} \phi^{\mu}-\phi^{\dagger \mu} \phi\right),
$$

and a corresponding conserved global charge or particle number

$$
N \equiv \int j^{0}|g|^{1 / 2} d x^{1} d x^{2} d x^{3},
$$

where $|g|$ is the absolute value of the determinant of the metric $g_{\mu \nu}$. A nonzero charge $N$ implies a time-varying scalar field by the definition of the current in Eq. (2); this time dependence is given by

$$
\phi(r, t)=\frac{1}{\sqrt{2}} \sigma(r) e^{-i \omega t},
$$

where the frequency $\omega$ is independent of position but varies with particle number $N$. The second requirement for the existence of soliton solutions is a constraint on the interaction potential $U\left(\sigma^{2}\right)$. For some forms of this potential free particles or a black hole will be energetically favored over a boson star for all values of $N$. However, if $U\left(\sigma^{2}\right)-1 / 2 m^{2} \sigma^{2}$ is negative for some range of $\sigma$, then stable boson-star solutions are guaranteed to exist for some values of $N$. The simplest polynomial form of the potential that satisfies this criterion is

$$
U=\frac{1}{2} m^{2} \sigma^{2}\left[1-\left(\frac{\sigma}{\sigma_{0}}\right)^{2}\right]^{2},
$$

which allows for a false vacuum within the boson star for $\sigma=\sigma_{0}$.

Having specified the form of the Lagrangian density $\mathfrak{Q}$, we can now determine the allowed boson-star solutions [17]. In the case of spherical symmetry, the spacetime metric of the boson star can be given in full generality by

$$
d s^{2}=-e^{2 u(r)} d t^{2}+e^{2 \bar{v}(r)} d r^{2}+r^{2}\left(d \theta^{2}+\sin ^{2} \theta d \phi^{2}\right) .
$$

The two metric functions $u(r)$ and $\bar{v}(r)$, along with the scalar-field configuration $\sigma(r)$ fully specify a particular boson-star solution. They are chosen to satisfy three independent, ordinary differential equations: the EulerLagrange equation for the scalar field, and the $t t$ and $r r$ components of the Einstein equation. In general these equations are stiff; the scalar field drops from $\sigma \simeq \sigma_{0}$ to zero across a surface layer of thickness $\mathrm{m}^{-1}$, while the metric functions vary over the much longer length scale $R \simeq G M$. If $\lambda \equiv \sigma_{0} / m_{\mathrm{Pl}} \ll 1$, the scalar field can be described by a step function

$$
\begin{array}{rlrl}
\sigma & =\sigma_{0} & r \leq R \\
& =0 & r>R .
\end{array}
$$

The system of equations then reduces to two coupled, firstorder equations for the metric functions interior to the boson-star surface at $r=R$. With the definitions

$$
\bar{r} \equiv \lambda^{2} m r \quad \text { and } \quad e^{-\bar{u}} \equiv \frac{\omega}{\lambda m} e^{-u},
$$

these two equations are

$$
\begin{aligned}
& 2 \bar{r} \frac{d \bar{v}}{d \bar{r}}=\left(\frac{1}{2} e^{-2 \bar{u}} \bar{r}^{2}-1\right) e^{2 \bar{v}}+1, \\
& 2 \bar{r} \frac{d \bar{u}}{d \bar{r}}=\left(\frac{1}{2} e^{-2 \bar{u}} \bar{r}^{2}+1\right) e^{2 \bar{v}}-1 .
\end{aligned}
$$

Physical boundary conditions imply that $\bar{v}(0)=0$, while $\bar{u}(0)$ and $R$ are chosen self-consistently to ensure that the metric function $u(r)$ smoothly matches onto the Schwarzschild solution $e^{2 u} \rightarrow(1-2 G M / r)$ for $r \geq R$. In this choice of coordinates $\bar{v}(r)$ need not be continuous at $r=R$. The metric functions $e^{2 u(r)}$ and $e^{2 \bar{v}(r)}$ are depicted in Fig. 1.

The final result of this analysis is that for fixed values of $m$ and $\sigma_{0}$, the boson-star solutions constitute a oneparameter family corresponding to different values of the particle number $N$. The boson-star mass $M$ increases monotonically with $N$, and the boson star becomes increasingly compact. Eventually a critical limit of compactness $R=2.869 G M$ is reached beyond which the boson star collapses into a black hole. We choose to investigate EMRIs into this critically compact boson star. We set $m=$ $\sigma_{0}$ to the value that yields the desired SMBS mass $M$. For the nontopological soliton considered here, $M \sim m_{\mathrm{Pl}}^{4} / m^{3}$, 


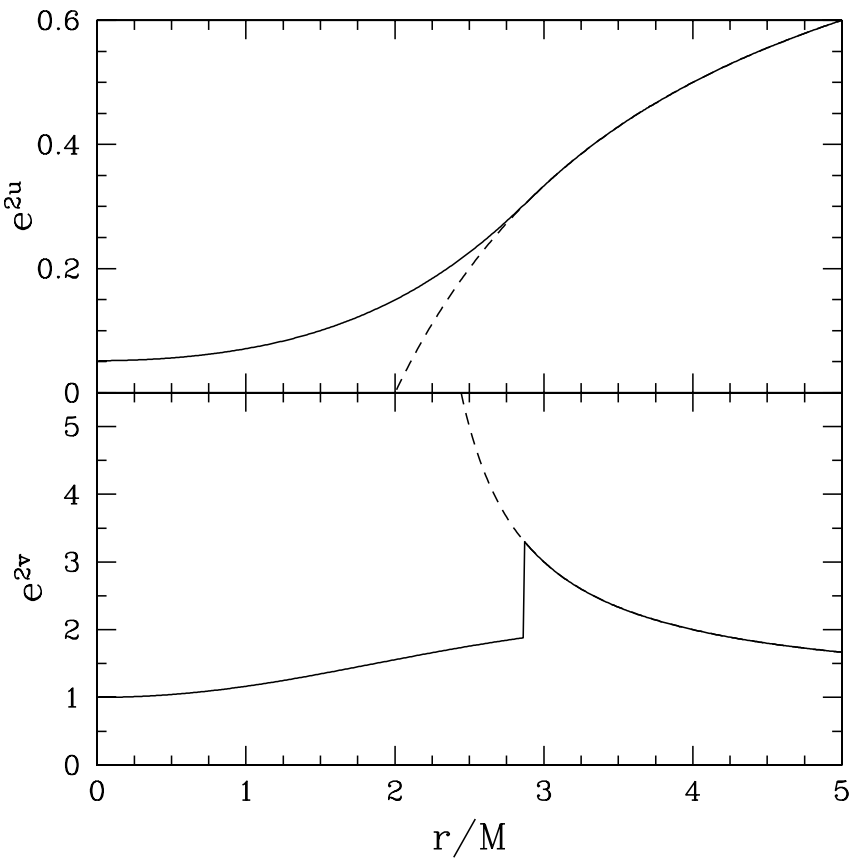

FIG. 1. The metric functions $e^{2 u(r)}$ and $e^{2 \bar{v}(r)}$ as functions of radius. The solid curves correspond to a boson star with $R=$ 2.869M while the dashed curves are for a black hole of the same mass. Outside the boson-star surface the two curves are identical, while for $r<R$ the solid curves are a numerical solution to Eq. (9) and the dashed curves are the Schwarzschild metric functions $e^{2 u(r)}=(1-2 G M / r), e^{2 \bar{v}(r)}=(1-2 G M / r)^{-1}$.

implying that a boson of mass $m \sim 10 \mathrm{TeV}$ will lead to a SMBS of $M \sim 10^{6} M_{\odot}$. The SMBS mass scales differently with $m$ for other types of boson stars.

\section{GEODESICS OF THE BOSON-STAR METRIC}

In the extreme-mass-ratio limit $\eta \equiv \mu / M \ll 1$, where $\mu$ and $M$ are the mass of the $\mathrm{CO}$ and boson star, respectively, the $\mathrm{CO}$ will travel along geodesics of the boson-star metric. During the course of the EMRI, energy and angular momentum are radiated away on time scales much longer than an orbital period. We can therefore make the adiabatic approximation that the $\mathrm{CO}$ will migrate smoothly between geodesics characterized by decreasing energy and angular momentum. A thorough description of the possible geodesics of the boson-star metric is thus essential to understanding the evolution of the CO's trajectory. This description can be simplified by noting that the metric of Eq. (6) is independent of $t$ and $\phi$, implying the existence of timelike and azimuthal Killing fields $\xi^{\mu}$ and $\psi^{\mu}$. For a CO traveling along a geodesic with four-velocity $u^{\nu}=$ $(d t / d \tau, d r / d \tau, d \theta / d \tau, d \phi / d \tau)$, the inner product of $u^{\nu}$ with a Killing field is conserved [18]. This allows a formal definition of the conserved energy and angular momentum per unit mass,

$E \equiv-g_{\mu \nu} \xi^{\mu} u^{\nu}=e^{2 u} \frac{d t}{d \tau}, \quad L \equiv g_{\mu \nu} \psi^{\mu} u^{\nu}=r^{2} \frac{d \phi}{d \tau}$.
Note that we can restrict ourselves to orbits with $\theta=\pi / 2$ without loss of generality because of spherical symmetry, in which case $d \theta / d \tau=0$. The definitions of Eq. (10), coupled with the norm of the four-velocity

$$
g_{\mu \nu} u^{\mu} u^{\nu}=-e^{2 u}\left(\frac{d t}{d \tau}\right)^{2}+e^{2 \bar{v}}\left(\frac{d r}{d \tau}\right)^{2}+r^{2}\left(\frac{d \phi}{d \tau}\right)^{2}=-1,
$$

provide three coupled, first-order differential equations that can be solved for the CO's orbit $\{t(\tau), r(\tau), \phi(\tau)\}$ as a function of proper time $\tau$.

While this approach formally solves the problem of geodesic motion, further insight can be gained by recasting Eq. (11) to make an analogy with one-dimensional particle motion [19],

$$
\begin{aligned}
E^{2} & =e^{2 u+2 \bar{v}}\left(\frac{d r}{d \tau}\right)^{2}+e^{2 u}\left(\frac{L^{2}}{r^{2}}+1\right) \\
& =e^{2 u+2 \bar{v}}\left(\frac{d r}{d \tau}\right)^{2}+V^{2}(r)
\end{aligned}
$$

The first term on the right-hand side of Eq. (12) acts like a positive-definite kinetic energy, while the second term is the potential well in which the one-dimensional particle motion occurs. This analogy is not as complete as in the case of a Schwarzschild black hole, where the metric functions conspire to make the coefficient of the kinetic term independent of position. For boson stars the term $e^{2 u+2 \bar{v}}$ acts like a position-dependent mass; while this will affect the particle motion quantitatively, it will not preclude the existence of bound orbits in the boson-star interior.

Plots of the effective potential $V(r)$ for different values of the angular momentum $L$ are given in Fig. 2. The effective potential per unit mass and ratio $r / M$ are dimensionless in units where $G=c=1$. Outside of the bosonstar surface at $R=2.869 M, V(r)$ is identical to that of Schwarzschild black holes. For $L^{2} \geq 12 M^{2}$, a local minimum exists at $r_{1} \geq 6 M$. Test particles with $E=V\left(r_{1}\right)$ can follow stable circular orbits at $r_{1}$, while those with slightly larger energies experience radial oscillations about this minimum corresponding to eccentric orbits. Particles with $E>1$ are unbound, and will either escape to infinity or, if they have energies greater than the local maximum and $d r / d t<0$, penetrate to the boson-star interior. It is here that the story changes dramatically from that of the black hole, where $V(r)$ plunges to negative infinity at the singularity. Particles that enter the black hole's event horizon are directed inexorably to the center, never to return. By contrast, the potential well of the boson star has finite depth, implying that for $L>0$ the effective potential is repulsive at short distances. This creates a second minimum in the effective potential at $r_{2}$ at which stable circular orbits and associated eccentric orbits can occur. Such orbits exist even for $E>1$, though it is unclear in what astrophysical context a CO might find itself on such a geodesic. 


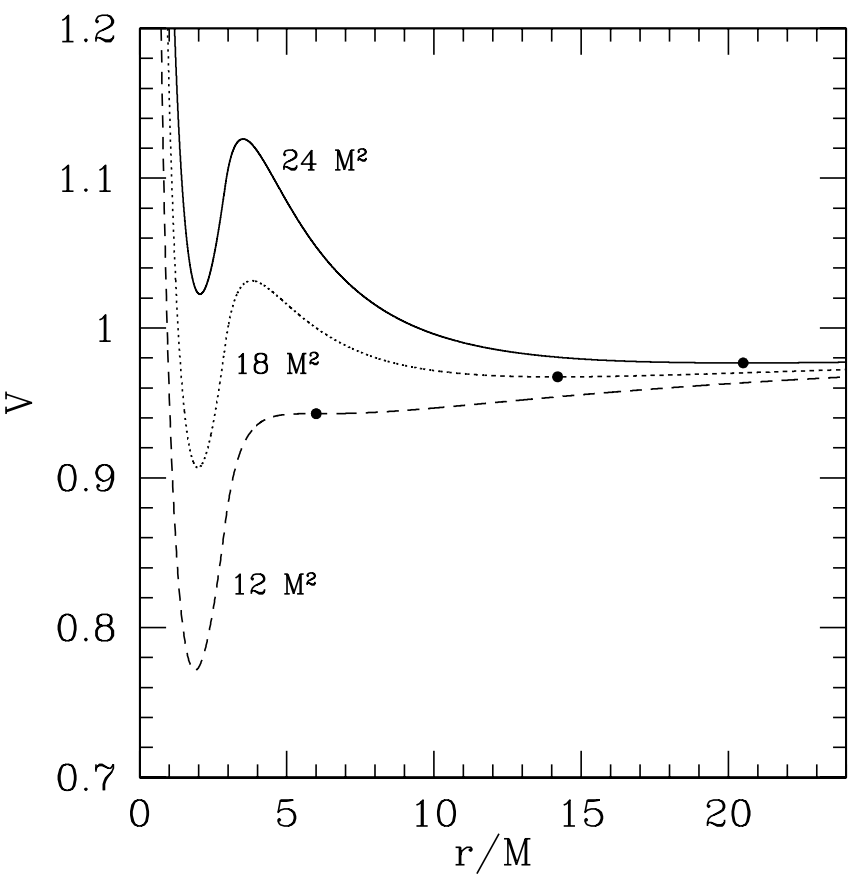

FIG. 2. The effective potential $V(r)$ of the boson star for three different values of the angular momentum. The solid, shortdashed, and long-dashed curves have $L^{2}=24 M^{2}, 18 M^{2}$, and $12 M^{2}$, respectively. The large black dots on each curve show the location of the outer minimum. For $L^{2}=12 M^{2}$, the lowest angular momentum for which there are two minima, the black dot is located at the innermost stable circular orbit $r=6 M$.

For a typical EMRI, a $\mathrm{CO}$ will be scattered onto an eccentric orbit and slowly circularize and wander inward as energy and angular momentum are lost to gravitational radiation. Eventually, either before or after circularization depending on the initial eccentricity and semimajor axis, the $\mathrm{CO}$ will reach the ISO and plunge into the boson-star interior. At this point the trajectory and corresponding gravitational waveform will diverge radically from the case of an EMRI into a black hole. Provided there is no nongravitational interaction between the $\mathrm{CO}$ and the scalar field, the inspiral will continue on a highly eccentric geodesic about the inner minimum of the effective potential. Energy and angular momentum will evolve continuously through the plunge, while both the apocenter and pericenter will change on orbital time scales as the center of radial oscillations changes from the outer minimum $r_{1}$ to the inner minimum $r_{2}$. High eccentricity at the ISO leads to even more complicated inspirals as the plunge takes place in a two-step process. The $\mathrm{CO}$ crosses over the local maximum of the effective potential several orbits before settling into the inner local minimum. The pericenter penetrates deep into the boson-star interior in the first stage of this two-step plunge, while in the second stage the apocenter shifts from outside to inside the local maximum. This inspiral would undoubtedly produce a very distinctive waveform, but only a quantitative analysis can determine whether such scenarios are possible. To make such a quantitative analysis we must first develop a suitable approximation for the calculation of the gravitational-wave emission.

\section{GRAVITATIONAL RADIATION}

Gravitational-wave emission by arbitrary sources is a challenging unsolved problem. In any asymptotically flat spacetime one can define the GW field,

$$
\bar{h}^{\alpha \beta} \equiv-(-g)^{1 / 2} g^{\alpha \beta}+\eta^{\alpha \beta},
$$

where $g_{\alpha \beta}$ is the metric, $g$ is its determinant, and $\eta^{\alpha \beta}$ is the flat-space Lorentz metric [20]. Far from the source, this quantity reduces to the trace-reversed metric perturbation $\bar{h}^{\alpha \beta}=h^{\alpha \beta}-1 / 2 \eta^{\alpha \beta} h$. The perturbation $\bar{h}^{\alpha \beta}$ satisfies the de Donder gauge condition

$$
\bar{h}^{\alpha \beta}{ }_{\beta}=0,
$$

and the exact Einstein field equations

$$
\square \bar{h}^{\alpha \beta}=-16 \pi \tau^{\alpha \beta} .
$$

We see that the GW field is sourced by an "effective stressenergy tensor" $\tau^{\alpha \beta}$ of the form [20]

$$
\begin{aligned}
\tau^{\alpha \beta}= & (-g)\left(T^{\alpha \beta}+t_{\mathrm{LL}}^{\alpha \beta}\right) \\
& +(16 \pi)^{-1}\left[\bar{h}^{\alpha \mu}{ }_{, \nu} \bar{h}^{\beta \nu}{ }_{, \mu}-\bar{h}^{\alpha \beta},{ }_{\mu \nu} \bar{h}^{\mu \nu}\right],
\end{aligned}
$$

where $T^{\alpha \beta}$ is the true stress-energy tensor and $t_{\mathrm{LL}}^{\alpha \beta}$, the Landau-Lifschitz pseudotensor given by Eq. (20.22) of [21], is a highly nonlinear function of the full metric. We thus see that for highly relativistic, strong-field sources the GW field $\bar{h}^{\alpha \beta}$ will itself provide a non-negligible contribution to its source $\tau^{\alpha \beta}$. In general Eq. (15) will lead to a system of tightly coupled, nonlinear differential equations that can only be integrated for brief periods numerically before becoming unstable. To make any analytical progress we must rely on a series of simplifying approximations of varying degrees of validity.

The first approximation we make is that in the extreme-mass-ratio limit $\eta \equiv \mu / M \ll 1$, the boson star is at rest at the origin of our coordinate system and its metric is static. The inspiraling $\mathrm{CO}$ is thus the sole time-varying source that contributes to GW emission. In reality the $\mathrm{CO}$ should raise tides on the surface of the boson star, which could then generate GWs themselves as well as back-react on the $\mathrm{CO}$, altering its trajectory. In an inspiral into a black hole, the energy loss due to the tidal interaction is at greatest only a few percent of the total flux, although this can still lead to several hundred cycles of phase difference in the gravitational waveform [22]. In the boson-star case the nature of the tidal interaction will be different and model-dependent, and provides another way to identify boson-star inspirals. However, the tidal interaction should still be a lesser effect than the orbital dynamics. The back- 
reaction on the orbit is suppressed by an additional factor of $\eta$, implying that for EMRIs with $\eta \simeq 10^{-6}$ it will introduce a very subdominant source of error into our calculations. The second approximation we make is to ignore the curvature induced by the boson star as it affects the propagation of GWs to infinity. GWs propagating outwards from $r \lessgtr\left(\lambda^{2} M\right)^{1 / 3}$, where $\lambda$ is the GW wavelength, will be distorted and backscattered by the background curvature of the boson star [20]. The importance of this effect will depend on the inner boundary conditions, which should differ significantly from those of a black hole with an event horizon. We ignore this complication entirely by assuming that backscattering does not occur. A third approximation that we make is to compute waveforms using weak-field formulas. We wish to apply these to orbits that come very close to the boson star, a regime in which there is no natural flat-space coordinate system in which to evaluate the weak-field expressions. Our approach is to identify the Schwarzschild coordinates $(t, r, \theta, \phi)$ of the inspiral trajectory with true flat-space spherical polar coordinates. This identification is exact for orbits out in the weak-field. There is no a priori reason why this identification is better than any other, e.g., identifying isotropic coordinates with the flat-space spherical polar coordinates. In practice, using different coordinate identifications gives different answers, but the differences are only a few percent. Taken together, these three approximations are equivalent to assuming the GWs from the EMRI will be the same as those that would be produced were the $\mathrm{CO}$ a "particle on a string," artificially constrained to move on an orbit $\{t(\tau), r(\tau), \phi(\tau)\}$ in flat space. In the case of blackhole inspirals, waveforms computed using this "hybrid" approach [23-25] have been found to compare quite well to waveforms computed using more accurate perturbative techniques.

In the flat-space, weak-field limit implied by the above approximations, the transverse traceless (TT) part of the GW field can be expanded in symmetric trace-free (STF) tensors [20],

$$
\begin{aligned}
h_{j k}^{\mathrm{TT}}= & {\left[\sum_{l=2}^{\infty}\left(\frac{4}{l !} r^{-1}\right)^{(l)} I_{j k A_{l-2}}(t-r) N_{A_{l-2}}\right.} \\
& \left.+\sum_{l=2}^{\infty}\left(\frac{8 l}{(l+1) !}\right) r^{-1} \epsilon_{p q(j}{ }^{(l)} S_{k) p A_{l-2}}(t-r) n_{q} N_{A_{l-2}}\right]^{\mathrm{TT}},
\end{aligned}
$$

where $A_{l} \equiv a_{1} \ldots a_{l}, I_{A_{l}}$, and $S_{A_{l}}$ are the rank- $l$ mass and current multipole-moment tensors, $n_{a}$ is a unit radial vector, and $N_{A_{l}} \equiv n_{a_{1}} \ldots n_{a_{l}}$. Parentheses on the indices denote taking the symmetric part, and a superscript $(l)$ in front of a tensor indicates taking the $l$ th time derivative. The energy and angular-momentum loss to gravitational radiation can also be expanded in multipole-moment tensors,

$$
\begin{aligned}
\frac{d(\mu E)}{d t}= & \sum_{l=2}^{\infty} \frac{(l+1)(l+2)}{(l-1) l} \frac{1}{l !(2 l+1) ! !}\left\langle{ }^{(l+1)} I_{A_{l}}^{(l+1)} I_{A_{l}}\right\rangle \\
& +\sum_{l=2}^{\infty} \frac{4 l(l+2)}{(l-1)} \frac{1}{(l+1) !(2 l+1) ! !} \\
& \times\left\langle{ }^{(l+1)} S_{A_{l}}^{(l+1)} S_{A_{l}}\right\rangle \\
\frac{d\left(\mu L_{j}\right)}{d t}= & \sum_{l=2}^{\infty} \frac{(l+1)(l+2)}{(l-1) l !(2 l+1) ! !}\left\langle\epsilon_{j p q}{ }^{(l)} I_{p A_{l-1}}^{(l+1)} I_{q A_{l-1}}\right\rangle \\
& +\sum_{l=2}^{\infty} \frac{4 l^{2}(l+2)}{(l-1)(l+1) !(2 l+1) ! !} \\
& \times\left\langle\epsilon_{j p q}{ }^{(l)} S_{p A_{l-1}}^{(l+1)} S_{q A_{l-1}}\right\rangle .
\end{aligned}
$$

Here \langle\rangle denotes averaging over an entire orbital period. A $\mu$ appears on the left-hand side of the equation in accordance with our definition of $E$ and $L$ as the energy and angular momentum per unit mass. This multipole-moment expansion is valid in principle for sources moving at arbitrarily relativistic velocities. We now further approximate that the sources are Newtonian; the maximum velocity is only mildly relativistic, and the internal stresses are small compared to the energy density [20]. Although this approximation is violated during the EMRI in the vicinity of the plunge, key features of the relativistic motion are preserved by constraining the $\mathrm{CO}$ to travel along geodesics that are exact even in the relativistic regime. Empirically, expansions in the post-Newtonian parameter $(L / \lambda)^{2} \sim v^{2}$ are found to give physical results (no outspiral) even in regions where they do not converge [26]. Assuming Newtonian sources, the mass and current multipole tensors are given by [20]

$$
\begin{gathered}
I_{A_{l}}=\left[\int \rho X_{A_{l}} d^{3} \vec{x}\right]^{\mathrm{STF}}, \\
S_{A_{l}}=\left[\int\left(\epsilon_{a_{l} p q} x_{p} \rho v_{q}\right) X_{A_{l-1}} d^{3} \vec{x}\right]^{\mathrm{STF}},
\end{gathered}
$$

where $X_{A_{l}} \equiv x_{a_{1}} \ldots x_{a_{l}}$, and $\rho$ is the energy density. In keeping with our assumption that the static boson-star metric does not contribute to the production of GWs, the energy density is simply that of the $\mathrm{CO}$,

$$
\rho(\vec{x})=\mu \delta^{3}\left(\vec{x}-\vec{x}_{\mathrm{CO}}(t)\right),
$$

where $\vec{x}_{\mathrm{CO}}(t)$ is the flat-space trajectory of the CO. The mass and current multipole moments $I_{\mathcal{A}_{\mathcal{L}}}$ and $S_{\mathcal{A}_{\mathcal{L}}}$ contribute to the GW field $h_{j k}^{\mathrm{TT}}$ at order $(M / r)(L / \lambda)^{l}$ and $(M / r)(L / \lambda)^{l+1}$, respectively, while the assumption of Newtonian sources induces errors of order $(L / \lambda)^{2}$ to each term [20]. It is therefore inconsistent to include terms higher than the mass quadrupole, current quadrupole, and mass octupole under this assumption. We consider only the mass-quadrupole term, relegating an analytic calculation of the error associated with this approximation to the 
appendix. In this case, Eqs. (17)-(19) reduce to the familiar form of the "quadrupole approximation"

$$
\begin{gathered}
h_{j k}^{\mathrm{TT}}=\frac{2}{r} \frac{d^{2} I_{j k}}{d t^{2}}, \\
\frac{d(\mu E)}{d t}=\frac{1}{5}\left\langle\frac{d^{3} I_{j k}}{d t^{3}} \frac{d^{3} I_{j k}}{d t^{3}}\right\rangle, \\
\frac{d\left(\mu L_{j}\right)}{d t}=\frac{2}{5}\left\langle\epsilon_{j p q} \frac{d^{2} I_{p k}}{d t^{2}} \frac{d^{3} I_{q k}}{d t^{3}}\right\rangle .
\end{gathered}
$$

How can the humble quadrupole approximation be justified for the eccentric, highly relativistic final stages of an EMRI into a boson star? While the energy and angularmomentum fluxes are indeed only approximate, the derivatives relating these fluxes to changes in the orbital parameters are exact, as are the equations of motion used in performing the orbital averages of Eqs. (24) and (25). Unlike direct post-Newtonian expansions for the time evolution of orbital elements, this "hybrid approximation" incorporates the exact orbital dynamics of geodesic motion $[23,27]$. This approach is self-consistent in the sense that the energy and angular momentum carried away by gravitational waves (within our quadrupole approximation) is equal to the loss of angular momentum and energy of the orbit. This approach can reproduce features missing from direct post-Newtonian expansions [which conserve energy and angular momentum only to $\left.\mathcal{O}\left(v^{2}\right)\right]$ such as the increase in orbital eccentricity just prior to plunge. Moreover, using exact geodesics in the flat-space quadrupole formula ensures that the fundamental frequencies of the orbit are reproduced in the gravitational waveforms. Although the distribution of power between harmonics is not correct, these approximate waveforms do encode the same orbital dynamics as the true waveform and therefore the qualitative features of inspiral waveforms should be well represented. At substantial computational expense, gravitational perturbation theory can calculate waveforms at infinity using the Teukolsky-Sasaki-Nakamura (TSN) formalism $[28,29]$. Such calculations for EMRIs in the Schwarzschild spacetime show agreement with the hybrid approach to within $5 \%$ to $45 \%$ for the time derivatives of the orbital semi-latus rectum and eccentricity even for moderate eccentricity $(e \sim 0.4)$ at $r \simeq 7 M[23,24]$. We hope that such accuracy will be retained in the case of boson stars, sparing us for now the additional computational expense of a numerical TSN approach. The details of adapting this hybrid approximation to boson-star EMRIs is the subject of the next section.

\section{ORBITAL EVOLUTION}

In a spherically symmetric spacetime, conservation of angular momentum implies that geodesic motion will be confined to an orbital plane [30]. Without loss of generality, this plane can be chosen to be the equatorial plane $\theta=$ $\pi / 2$. In this case, the position of the $\mathrm{CO}$ is fully specified by a radius $r$ and a true longitude $\phi$. A geodesic will be characterized by orbital elements like the semi-latus rectum $p$ and eccentricity $e$, in terms of which the radius is given by

$$
r=\frac{p}{1+e \cos \psi} .
$$

The true anomaly $\psi$ can be specified independently of $\phi$, since in general the pericenter will precess. In the hybrid approximation, EMRI orbits and waveforms are calculated in a two-step process that hinges on the assumption of adiabaticity; i.e., orbital elements like $p$ and $e$ vary on time scales much longer than an orbital period over which $\psi$ and $\phi$ change [23,27]. This allows the differential equations governing the evolution of $p$ and $e$ to be integrated with a much longer time step than is required for evolving $\psi$ and $\phi$. We will first describe how the two stages of integration are accomplished with this assumption, and then we will discuss the corrections necessary to patch together a physically reasonable orbit through those regions where the assumption is violated.

In the first stage of our calculation, the EMRI's trajectory through a phase space of $p$ and $e$ is determined by relating these quantities to $E$ and $L$, and then using this relation and Eqs. (24) and (25) to obtain differential equations for $p$ and $e$. Particular values of $p$ and $e$ uniquely determine the pericenter $r_{p}=p /(1+e)$ and apocenter $r_{a}=p /(1-e)$ from Eq. (26). At both pericenter and apocenter, $d r / d \tau=0$ implying $V\left(r_{p}\right)=V\left(r_{a}\right)=E$ by Eq. (12). This equation can be solved to obtain the energy $E$ and $L$ as functions of $p$ and $e$,

$$
\begin{aligned}
& L(p, e)=\left[\left(e^{2 u\left(r_{p}\right)}-e^{2 u\left(r_{a}\right)}\right)\left(\frac{e^{2 u\left(r_{a}\right)}}{r_{a}^{2}}-\frac{e^{2 u\left(r_{p}\right)}}{r_{p}^{2}}\right)^{-1}\right]^{1 / 2}, \\
& E(p, e)=\left[e^{2 u\left(r_{a}\right)}\left(\frac{L^{2}}{r_{a}^{2}}+1\right)\right]^{1 / 2} .
\end{aligned}
$$

First-order differential equations for the time evolution of $p$ and $e$ can then be derived from the exact derivatives of $E(p, e)$ and $L(p, e)$ along with the quadrupole approximations of Eqs. (24) and (25),

$$
\frac{d p}{d t}=\frac{\frac{d L}{d t}-\frac{\partial L / \partial e}{\partial E / \partial e} \frac{d E}{d t}}{\frac{\partial L}{\partial p}-\frac{\partial L / \partial e}{\partial E / \partial e} \frac{\partial E}{\partial p}}, \quad \frac{d e}{d t}=\frac{\frac{d L}{d t}-\frac{\partial L / \partial p}{\partial E / \partial p} \frac{d E}{d t}}{\frac{\partial L}{\partial e}-\frac{\partial L / \partial p}{\partial E / \partial p} \frac{\partial E}{\partial e}} .
$$

It is the combination of these exact derivatives with the quadrupole approximation for the fluxes that accounts for the superior performance of this hybrid approximation over the consistent (in that we include all terms up to a given order in $v^{2}$ ) post-Newtonian approach. The quadrupole fluxes themselves can be determined by inserting Eq. (22) for the CO's energy density into Eq. (20) for the Newtonian quadrupole moments. Using an overdot to denote a derivative with respect to coordinate time $t$, we find that the time derivatives of the quadrupole moment appearing in Eqs. (24) and (25) take the form 


$$
\begin{aligned}
\dddot{I}_{j k} \dddot{I}_{j k}= & 2 \eta^{2}\left[(1 / 3)(\dddot{r}+3 \ddot{r} \dot{r})^{2}+\left(6 \ddot{r} \dot{\phi} r+6 \dot{r} \ddot{\phi} r+6 \dot{r}^{2} \dot{\phi}-4 \dot{\phi}^{3} r^{2}+\dddot{\phi} r^{2}\right)^{2}+\left(\dddot{r} r+3 \ddot{r} \dot{r}-12 \dot{r} \dot{\phi}^{2} r-6 \ddot{\phi} \dot{\phi} r^{2}\right)^{2}\right], \\
\epsilon_{j p q} \ddot{I}_{p k} \dddot{I}_{q k}= & 2 \eta^{2}\left[\left(\ddot{r} r+\dot{r}^{2}-2 r^{2} \dot{\phi}^{2}\right)\left(6 \ddot{r} \dot{\phi} r+6 \dot{r} \ddot{\phi} r+6 \dot{r}^{2} \dot{\phi}-4 \dot{\phi}^{3} r^{2}+\dddot{\phi} r^{2}\right)-\left(4 \dot{r} r \dot{\phi}+r^{2} \ddot{\phi}\right)\right. \\
& \left.\times\left(\dddot{r} r+3 \ddot{r} \dot{r}-12 \dot{r} \dot{\phi}^{2} r-6 \ddot{\phi} \dot{\phi} r^{2}\right)\right] .
\end{aligned}
$$

The derivatives appearing in Eq. (29) can be evaluated analytically by solving Eqs. (10) and (11) for the equations of motion

$$
\begin{gathered}
\frac{d t}{d \tau}=E e^{-2 u}, \\
\frac{d \phi}{d \tau}=\frac{L}{r^{2}}, \\
\left(\frac{d r}{d \tau}\right)=e^{-2 \bar{v}}\left[E^{2} e^{-2 u}-\left(\frac{L^{2}}{r^{2}}+1\right)\right],
\end{gathered}
$$

dividing Eqs. (31) and (32) by Eq. (30), and then taking the appropriate derivatives. We then perform the orbital average appearing in Eqs. (24) and (25),

$$
\langle f(r)\rangle=\frac{\int_{r_{p}}^{r_{a}} f(r) \frac{d r}{\dot{r}}}{\int_{r_{p}}^{r_{a}} \frac{d r}{\dot{r}}} .
$$

The products of these manipulations are first-order differential equations for $\dot{p}$ and $\dot{e}$ that can be integrated forward in time for arbitrary initial $p_{0}$ and $e_{0}$. This tabulated phasespace trajectory $\{p(t), e(t)\}$ will then be used in the second stage to produce the real-space trajectory $\{r(t), \phi(t)\}$.

The second stage of calculating the EMRI real-space trajectory is essentially no more complicated than integrating the equations of motion Eqs. (30)-(32) with a time step that is a sufficiently small fraction of the orbital period as to obtain the desired accuracy. The phase-trajectory determined in the first stage can be linearly interpolated to obtain $p(t)$ and $e(t)$, from which the energy and angular momentum follow from the relations $E(p, e)$ and $L(p, e)$. These are then inserted into the equations of motion along with $r$ and $\phi$ at the beginning of the time step. A minor technical difficulty arises for circular orbits $(e=0)$ and at the turning points of eccentric orbits $(\psi=n \pi)$ where the relation

$$
\frac{d \psi}{d \tau}=\frac{1+e \cos \psi}{e r \sin \psi} \frac{d r}{d \tau}
$$

becomes undefined. This problem can be solved by a judicious Taylor expansion of Eq. (32) about the appropriate $r$ values. Once the real-space trajectory $\{x(t), y(t), z(t)\}$ has been obtained, the quadrupole waveform of Eq. (23) can be calculated by taking the appropriate numerical derivatives. This portion of the calculation does not involve any special features of the boson-star spacetime, in keeping with the "particle on a string" approximation. The GW field $h_{i j}^{\mathrm{TT}}$ can then be decomposed into plus and cross polarizations by defining unit vectors in the plane of the sky. With $\hat{L}$ a unit vector parallel to the CO's angular momentum and $\hat{n}$ a unit vector pointed from the observer to the source, we define

$$
\hat{p} \equiv(\hat{n} \times \hat{L}) /|\hat{n} \times \hat{L}|, \quad \hat{q} \equiv \hat{n} \times \hat{p} .
$$

Note that the sign of $\hat{q}$ differs from that of Ref. [26], but in other respects our conventions are equivalent. Our basis vectors $\hat{p}$ and $\hat{q}$ are constants because $\hat{L}$ does not precess in a spherically symmetric spacetime. The two polarization basis tensors can be defined as

$$
H_{i j}^{+} \equiv \hat{p}_{i} \hat{p}_{j}-\hat{p}_{i} \hat{p}_{j}, \quad H_{i j}^{\times} \equiv \hat{p}_{i} \hat{q}_{j}+\hat{q}_{i} \hat{p}_{j},
$$

allowing the GW field to be expressed as two amplitudes,

$$
h_{i j}^{\mathrm{TT}}(t)=A^{+}(t) H_{i j}^{+}+A^{\times}(t) H_{i j}^{\times} .
$$

In the next section, we will examine the trajectories $\{x(t), y(t), z(t)\}$ and GW amplitudes $\left\{A^{+}(t), A^{\times}(t)\right\}$ for different EMRIs.

Before presenting these results, we must explain the kludges we have chosen where the hybrid approximation described above is no longer valid. Our philosophy is to adopt the simplest, least computationally intensive approach that yields physically reasonable waveforms. The numerous approximations involved in the "particle on a string" model introduce at least a $10 \%$ error into our trajectories and waveforms in the highly eccentric, relativistic limit $[23,24]$; to demand greater accuracy where the hybrid approximation is also violated would be inconsistent. Special provisions beyond the hybrid approximation must be made whenever $p$ and $e$ are changing on time scales shorter than the orbital period. For extremely eccentric orbits, gravitational radiation will tend to be emitted in bursts near pericenter, while the $\mathrm{CO}$ will be found near apocenter for most of the orbital period. This is particularly true of "zoom-whirl" orbits like those found around rapidly spinning black holes, where the CO may whirl for many radians near pericenter before zooming back out to apocenter [31]. In such cases, the orbital averaging of Eq. (33) will lead to an artificially smooth phase-space trajectory $\{p(t), e(t)\}$. For boson-star EMRIs, this adiabatic approximation is most flagrantly violated during the plunge itself. Since the rapidly varying true anomaly $\psi(t)$ is only calculated in the second stage of our two-step approach, the $\mathrm{CO}$ could be anywhere along its orbit at the time the plunge is calculated to occur in the first stage. This poses problems in both stages of the calculation. As described qualitatively in Sec. III, a CO initially oscillating about the outer minimum of the effective potential $V(r)$ can 
plunge into the boson star with $L^{2}>12 M^{2}$, in which case two minima will still exist even after the plunge. For a time, the energy of the $\mathrm{CO}$ will exceed $V_{\max }(L)$, the local maximum of $V(r)$, so that both of the local minima will lie between the orbit's pericenter and apocenter. Such a highly eccentric orbit will radiate energy very efficiently, implying that the $\mathrm{CO}$ will quickly plunge into the inner minimum. However, if the initial conditions were such that the $\mathrm{CO}$ began on a geodesic with $E$ was significantly above $V_{\max }$, there is no reason a priori why it could not first fall into the outer minimum before eventually inspiraling into the boson-star interior. Which minimum the $\mathrm{CO}$ ends up in depends on where the $\mathrm{CO}$ is in its orbit when $E$ drops below $V_{\max }$. But the true anomaly $\psi(t)$ is not calculated in the first stage of the hybrid approximation, so we have no way of accurately knowing which minimum to choose. The semilatus rectum $p$ and eccentricity $e$ change discontinuously when the $\mathrm{CO}$ falls into one of the two minima, a sure sign that the adiabatic condition no longer holds. We assume without rigorous proof that the $\mathrm{CO}$ always ends up in the inner minimum in such a situation. This assumption comes back to haunt us in the second stage, since the CO may well be near apocenter at the time determined for the plunge in the first stage. In this case, no possible instantaneous change in $\psi$ would allow $r$ to remain continuous. Instead, we fix $p$ and $e$ to their values at the time of the plunge, and allow the $\mathrm{CO}$ to travel along a geodesic until it reaches the local maximum at $r_{\max }$. This then becomes the apocenter of the CO's radial motion about the inner minimum, and we resume linearly interpolating $p$ and $e$ from the phasespace trajectory tabulated in the first stage. Having exhaustively described our technique, we will now examine the fruits of our labor.

\section{RESULTS}

First we consider the phase-space trajectories $\{p(t), e(t)\}$ generated in the first part of our calculation. We choose for our fiducial model a $3 M_{\odot} \mathrm{CO}$ spiraling into a $3 \times 10^{6} M_{\odot}$ boson star. Three different trajectories are depicted in Fig. 3, each with $p_{0}=10 M$ but with differing initial eccentricities $\left(e_{0}=0.0,0.4,0.8\right)$. Several features of the phase-space evolution are particularly striking. Outside the boson-star surface at $R=2.869 G M$, the spacetime is identical to that of a Schwarzschild black hole and the behavior of the EMRI is well known. Orbits far from the boson star rapidly circularize, but those that retain an appreciable eccentricity exhibit an increase in the eccentricity in the last few orbits before the ISO. This behavior reflects the increasing shallowness of the outer minima as the ISO is approached, and is not captured by post-Newtonian expansions that ignore geodesic motion [23,31]. The second important feature is the discontinuity in each of the three trajectories as the plunge is reached. As described in the previous section, the actual position of the $\mathrm{CO}$ remains continuous; only the orbital elements $p$ and $e$ change at the
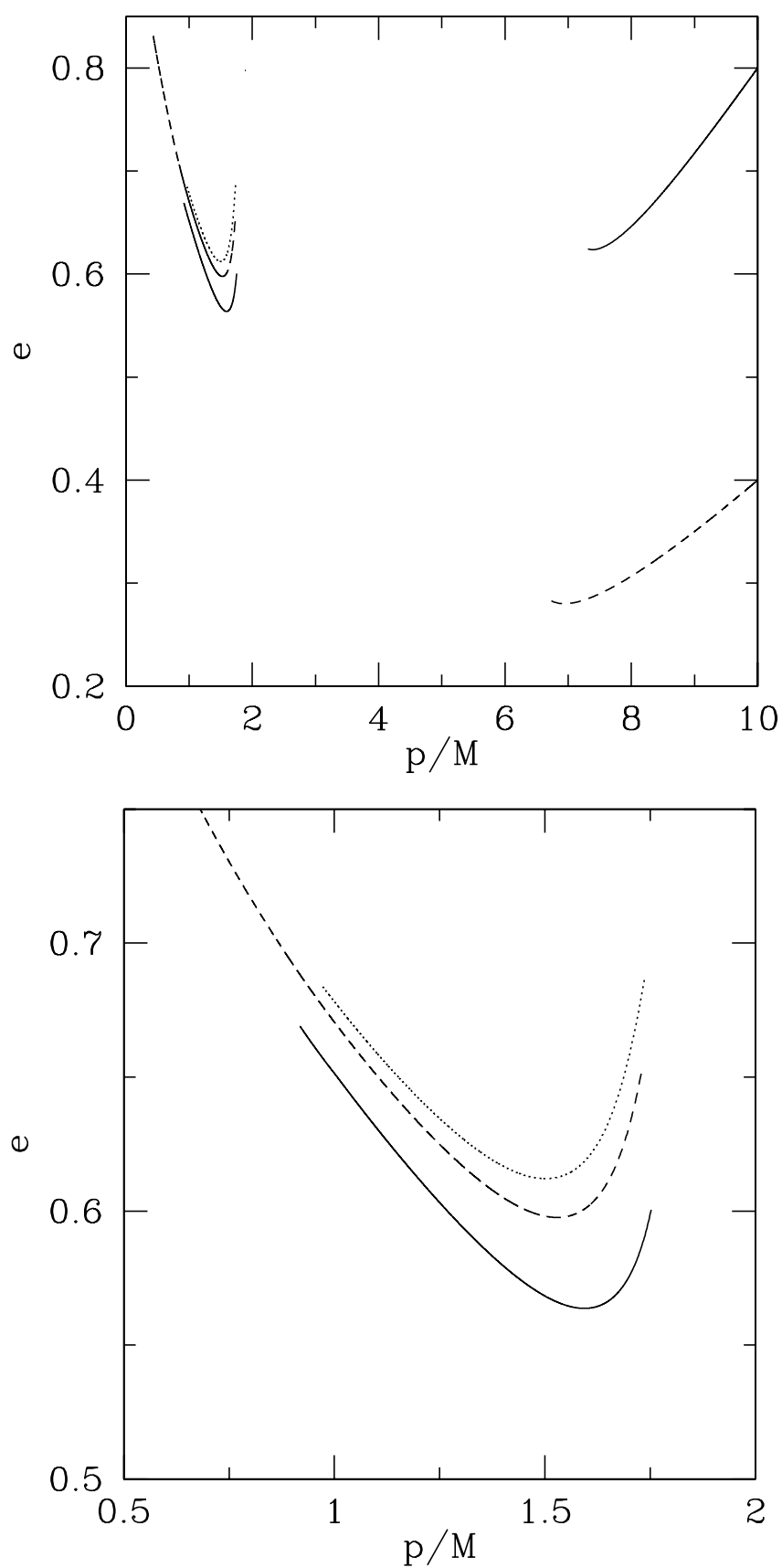

FIG. 3. The phase-space trajectories $\{p(t), e(t)\}$ for three different EMRIs into a supermassive boson star. The solid, dashed, and dotted curves begin with eccentricities $e_{0}=0.8,0.4$, and 0.0 , respectively. The top panel shows the complete EMRI from $p_{0} / M=10.0$, while the lower panel is a close-up of the postplunge phase of the EMRI that appears in the upper-left corner of the top panel. In both panels time increases from right to left as $p / M$ decreases monotonically.

instant of the plunge. This is accomplished by an appropriate instantaneous change in the true anomaly $\psi$. For the EMRIs with $e_{0}=0.8$ and 0.4 , the orbit is actually divided into three distinct pieces, with the middle section belonging to geodesics where both minima lie between pericenter 
and apocenter. This portion cannot be seen in Fig. 3 because it only lasts for a few orbital periods, which is short compared to the time scales on which $p$ and $e$ are changing. It is interesting to note that the three curves have reversed their order in the plunge; the more eccentric the EMRI prior to plunge, the lower its eccentricity afterwards. This can be understood by realizing that orbits with higher residual eccentricity plunge into the boson star with higher angular momentum $L$, implying that the inner minimum will be much steeper as depicted in Fig. 2. The pericenter and apocenter will be closer together in this narrow potential well, leading to a correspondingly smaller post-plunge eccentricity. A final surprising feature of these EMRIs is the sharp increase in eccentricity for the final stages of the EMRI deep within the interior of the boson star. The energy and angular momentum are monotonically decreasing during this stage of the EMRI in keeping with GWinduced losses; only the relations $E(p, e)$ and $L(p, e)$ are unusual. As angular momentum is lost, the narrow inner minimum rapidly broadens, leading to a greater separation between pericenter and apocenter. The separation

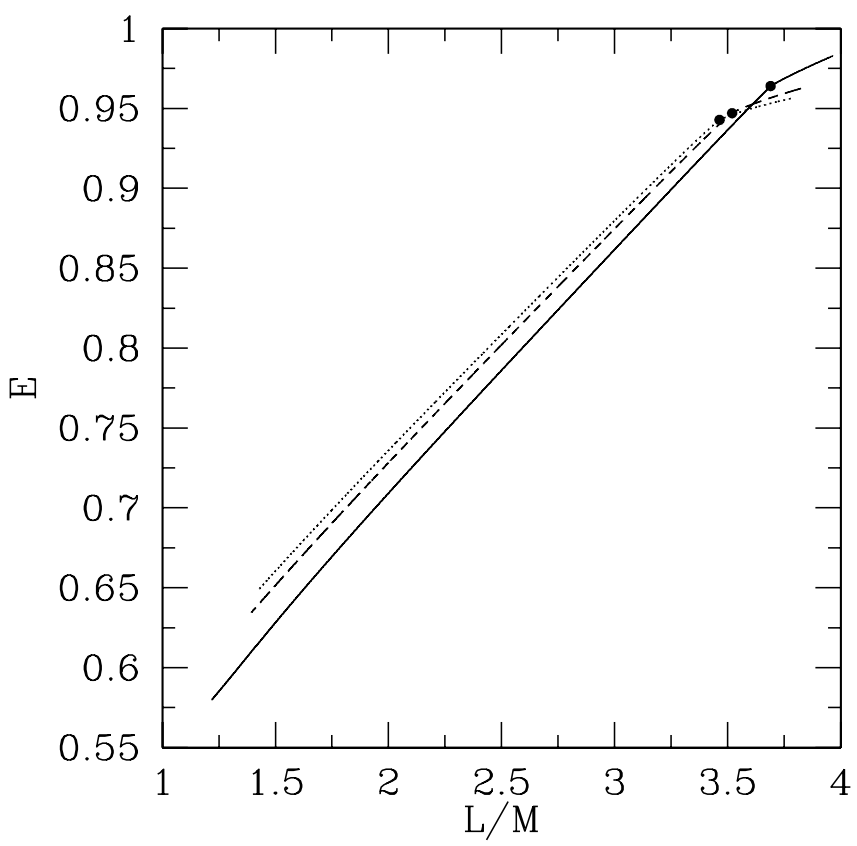

FIG. 4. The phase-space trajectories $\{L(t), E(t)\}$ for the three EMRIs depicted in Fig. 3. As before, the solid, dashed, and dotted curves begin with eccentricities $e_{0}=0.8,0.4$, and 0.0 , respectively. The large black dots correspond to the points at which the COs plunge into the boson star. Note that the curves cross each other, implying that two different geodesics can be characterized by the same energy and angular momentum. This feature, unique to the boson-star case, follows from the existence of bound geodesics both interior and exterior to the local maximum of the effective potential for certain energy and angular momenta. No such crossing appears in Fig. 3 as $p$ and $e$ do uniquely specify a given geodesic.

$$
r_{a}-r_{p}=\frac{2 p e}{1-e^{2}}
$$

is a sharply increasing function of eccentricity, explaining why the broadening inner minimum leads to rising eccentricity. For completeness, a plot of these same trajectories in the phase space of angular momentum and energy $\{L(t) / M, E(t)\}$ is given in Fig. 4. Note that $E$ and $L$ are continuous at the plunge as is required for physical quantities.

We now consider the real-space trajectory $\{x(t), y(t), z(t)\}$ itself. Choosing the orbital and equatorial planes to coincide, $z=0$. Several orbits of an initially circular EMRI on both sides of the plunge are shown in Fig. 5. The trajectory remains continuous during the plunge, and the pericenter precesses by an appreciable fraction of a radian on each post-plunge orbit. Of more interest is the EMRI of Fig. 6 with $e_{0}=0.4, p_{0}=10.0 M$. We have omitted the EMRI with $e_{0}=0.8$ because the two initially eccentric EMRIs appear qualitatively similar. Both the $e_{0}=0.4$ and $e_{0}=0.8$ EMRIs experience a two-step plunge as described qualitatively in Sec. III. In the first stage, the pericenter migrates deep into the interior of the boson star. Only several orbits later does the apocenter finally move inward of the local maximum. While twostep plunge may not be immediately apparent from the real-space trajectory, it leaves a very distinctive signature in the gravitational waveform.

First we consider the waveform of the initially circular EMRI as shown in Fig. 7. The system is viewed at an inclination angle of $45^{\circ}$ at a distance of $10 \mathrm{Mpc}$. The two

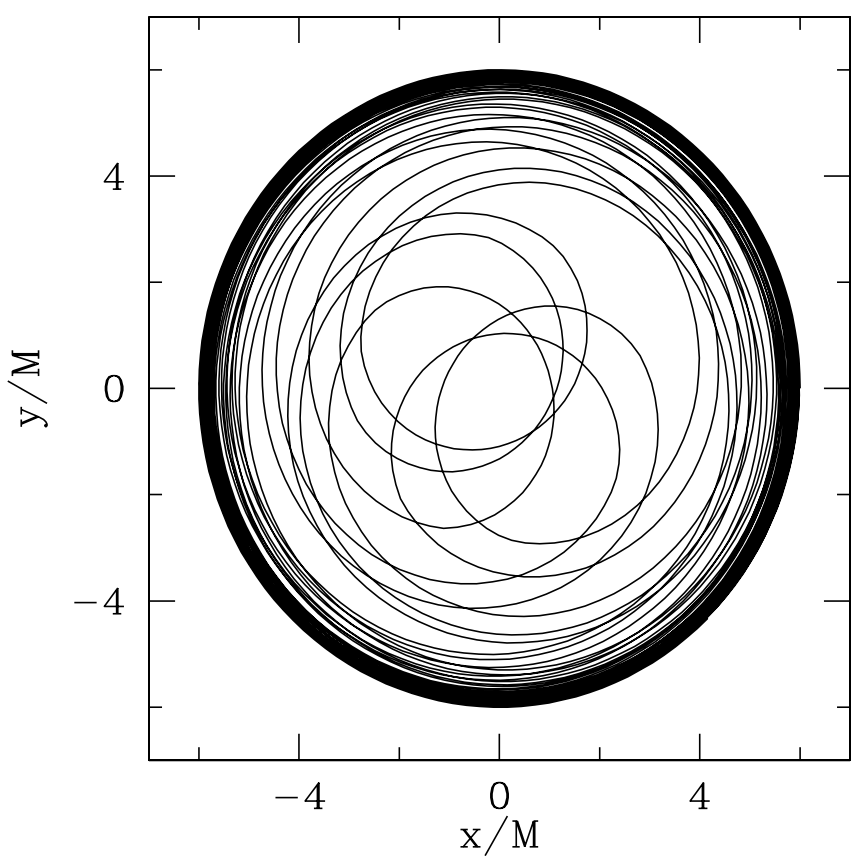

FIG. 5. The real-space trajectory $\{x(t), y(t)\}$ for an initially circular EMRI. For purposes of clarity, only a period of approximately $150000 \mathrm{~s}$ in the vicinity of the plunge is depicted. 

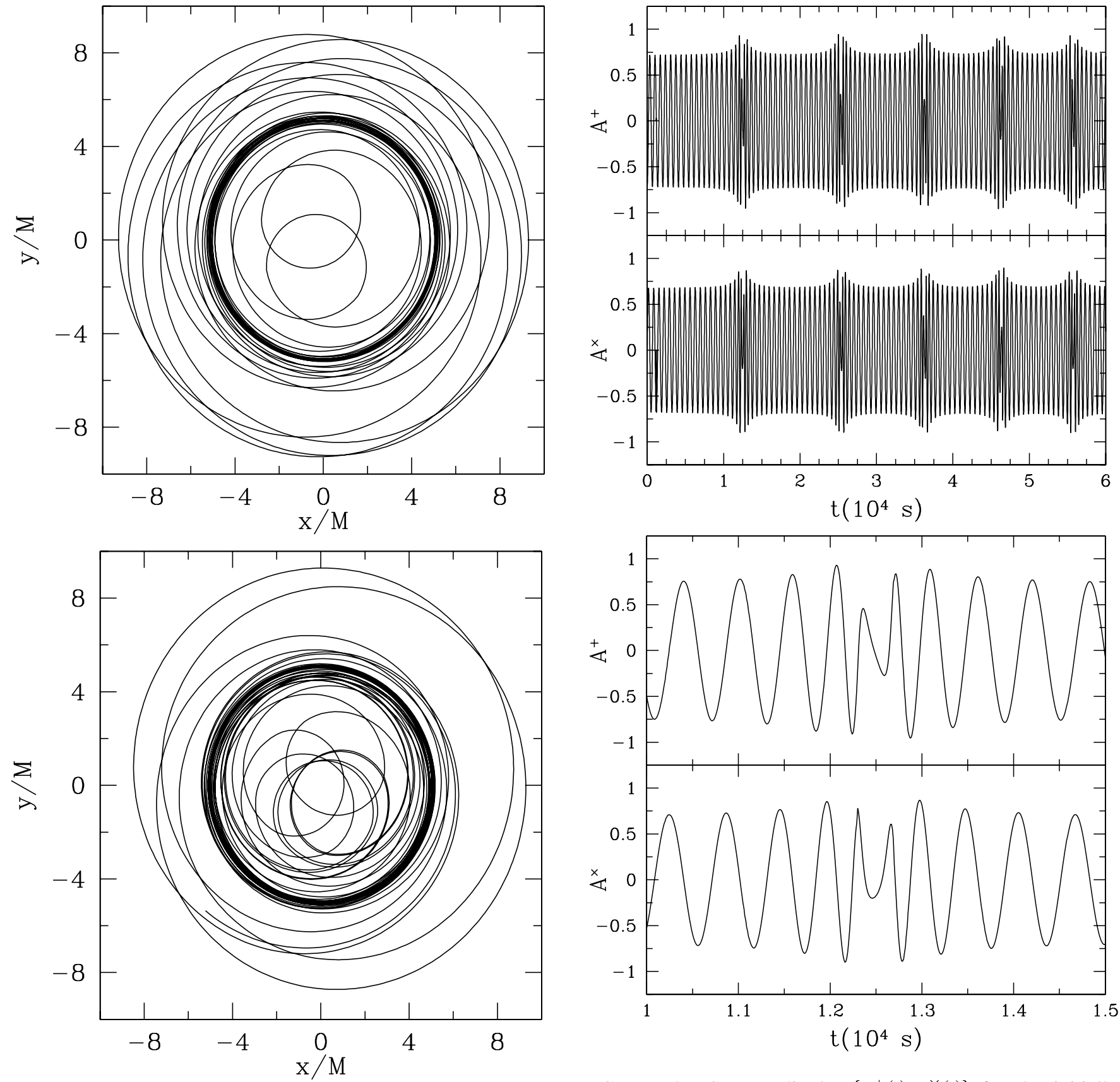

FIG. 7. The GW amplitudes $\left\{A^{+}(t), A^{\times}(t)\right\}$ for the initially circular EMRI depicted in Fig. 5. The dimensionless amplitudes are given in units of $10^{-20}$, while the time is in units of $10^{4} \mathrm{~s}$. The upper panel shows $60000 \mathrm{~s}$ of the inspiral, including the plunge at $t=0.12 \times 10^{4} \mathrm{~s}$. The lower panel zooms in on the portion of the waveform produced in the interior of the boson star itself.

polarizations provide similar information because the given decomposition depends on our arbitrary choice of basis tensors. Before the plunge, the waveform is identical to that of a circular inspiral into a Schwarzschild black hole. The fundamental oscillation has a period of about $500 \mathrm{~s}$, one-half the azimuthal period over which $\phi$ changes by $2 \pi$ radians. Since the orbit is circular, the amplitude only varies on the extremely long time scale on which

energy and angular momentum are lost. After the plunge, the eccentricity increases sharply and we see an amplitude modulation with a period of about $10000 \mathrm{~s}$. This corresponds to radial oscillations about the inner minimum within the boson star. The amplitude creeps upwards as the $\mathrm{CO}$ approaches pericenter with increasing acceleration. Note, however, the peculiar feature at pericenter itself, 
where the amplitude should be greatest. Instead, the cycle at pericenter is suppressed because once the $\mathrm{CO}$ crosses the boson-star surface, it is only accelerated by the mass interior to its position. We present a close-up of this distinctive feature in the bottom panel of Fig. 7.

The waveform of the initially eccentric EMRI as shown in Fig. 8 is even more unique. All three phases of the EMRI can be easily distinguished by the naked eye. In the first phase, from the beginning of the waveform until the first step of the plunge at $t=1.4 \times 10^{4} \mathrm{~s}$, the CO moves on ordinary eccentric geodesics of Schwarzschild. Amplitude modulation corresponding to oscillations about the outer minimum are seen, the crests near pericenter are smooth because there are as yet no close approaches to the boson star. In the second phase, between the two steps of the plunge, the $\mathrm{CO}$ moves on geodesics with both minima between pericenter and apocenter. These geodesics are extremely eccentric, leading to longer radial oscillations and an approximate $50 \%$ increase in the period of the amplitude modulation. The apocenter remains nearly constant during the plunge, marked by the continued presence of deep troughs in the amplitude modulation. The pericenter, however, has migrated deep into the interior of the boson star as evidenced by the spikes on each crest produced during close approaches. The peculiar feature at pericenter itself is barely visible on this scale as a very narrow gap in these spikes. The second step of the plunge occurs at $t=8.0 \times 10^{4} \mathrm{~s}$, when the CO crosses over the

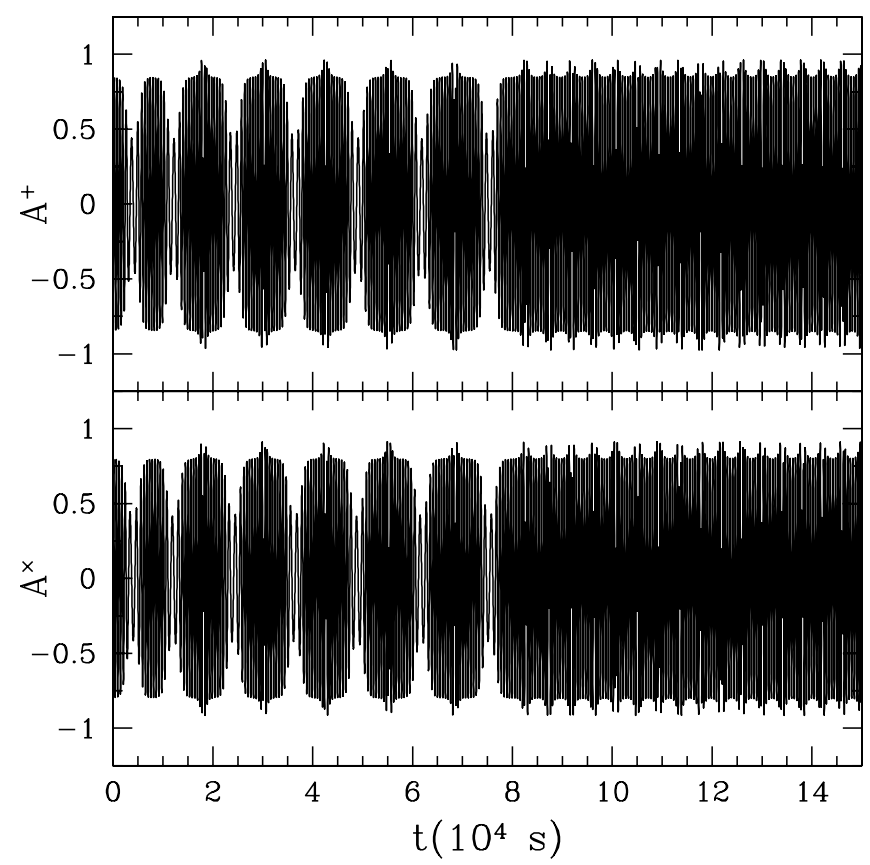

FIG. 8. The GW amplitudes $\left\{A^{+}(t), A^{\times}(t)\right\}$ for the initially eccentric EMRI depicted in Fig. 6. As in Fig. 7, the dimensionless amplitudes are given in units of $10^{-20}$, while the time is in units of $10^{4} \mathrm{~s}$. All three portions of the inspiral are clearly visible. local maximum for the final time. The second step is in some sense the opposite of the first, in that the pericenter remains constant while the apocenter plunges inwards. We see that the spikes marking pericenter remain unchanged between the second and third phases, but the deep troughs in the amplitude modulation from distant apocenters have
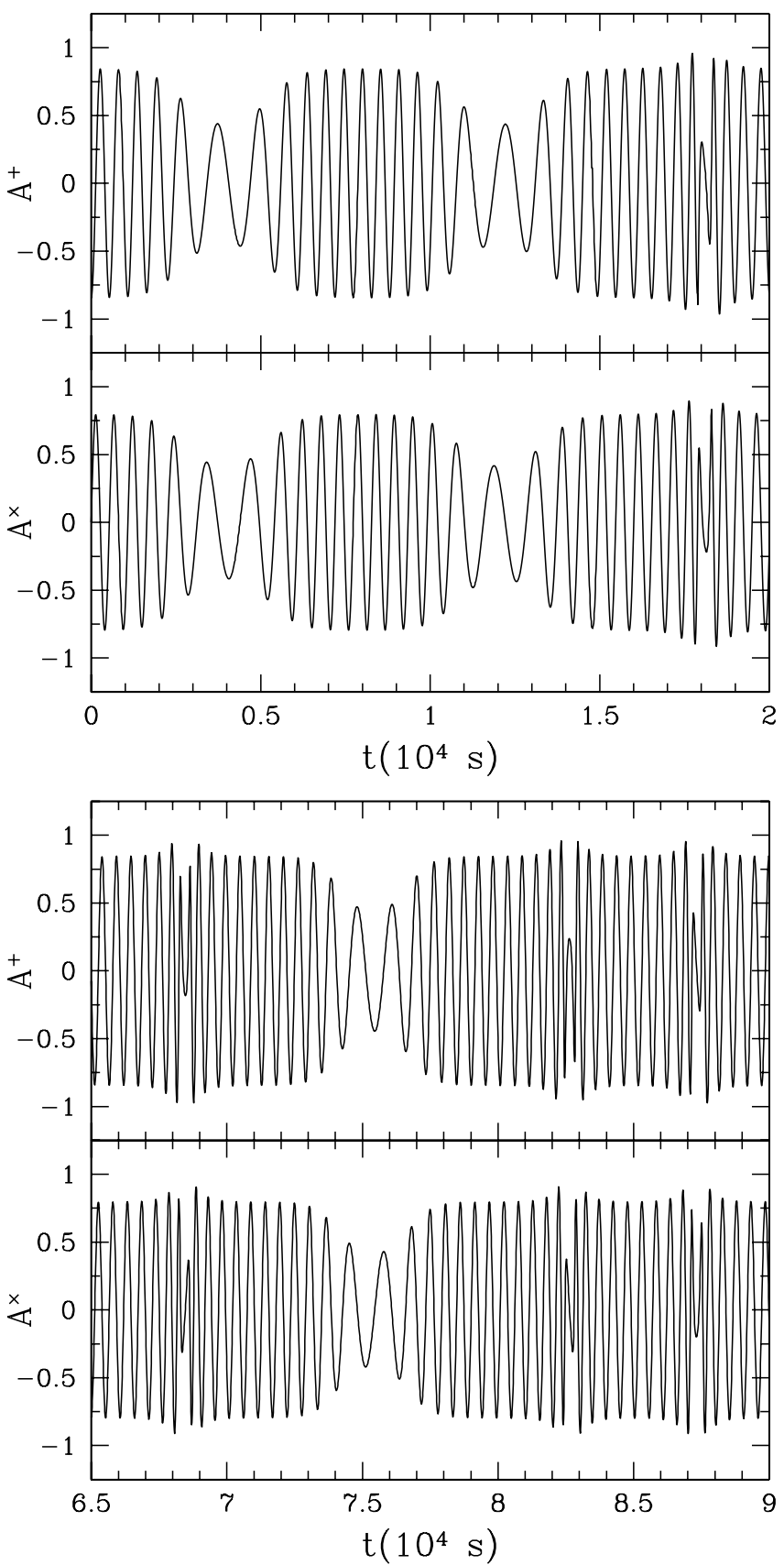

FIG. 9. Close-ups of the waveform shown in Fig. 8 for the initially eccentric EMRI. The top panel depicts the first step of the plunge; note that only the rightmost crest exhibits the peculiar feature produced in the boson-star interior. The bottom panel shows the second step of the plunge; note how different the final distant apocenter at $t=7.5 \times 10^{4} \mathrm{~s}$ appears from the apocenter at $t=8.5 \times 10^{4} \mathrm{~s}$. 
vanished. Close-ups of the two steps of the plunge are shown in Fig. 9.

\section{DISCUSSION}

Strong evidence supports the existence of supermassive compact objects at the centers of many if not most galaxies. Accretion onto these objects is presumed to power active galactic nuclei, and direct observations of velocity dispersions within galactic cusps reveal that a pointlike mass dominates the dynamics within $\sim 1 \mathrm{pc}$ from the center. While all this evidence is consistent with these objects being black holes, the "smoking-gun" signature of an event horizon has yet to be observed. Until such a definitive determination is made, other candidates such as boson stars should continue to be considered. Previous work has suggested that accretion-induced x-ray bursts might be an optical signature of boson stars [2]. We rely here on distinguishing boson stars from black holes through their gravitational effects. One possibility is their different properties as a strong gravitational lens; a shadow induced by a black hole's event horizon may soon be observed in Sag A* at submillimeter wavelengths [4]. In this paper, we have investigated the possibility that the inspiral of a several solar-mass $\mathrm{CO}$ into a boson star may produce a distinctive spectrum of GWs. LISA is conservatively expected to observe several such inspirals each year with appreciable signal-to-noise ratios $[9,10]$.

Previous work has examined the possibility of measuring the central object's multipole moments, and testing whether or not they satisfy the predictions of the "nohair" theorem for black holes [16]. This formalism has been applied, in particular, to boson stars with anomalously large mass-quadrupole moments, where an appreciable effect on the GW-induced loss of energy was discovered [13]. Here we have gone beyond such an analysis by calculating approximations to the waveforms that might actually be observed by LISA. The spherically symmetric boson stars we consider have Schwarzschild spacetimes outside their surfaces; a multipole analysis of GWs produced while the $\mathrm{CO}$ is still outside the boson star would not reveal any violations of the "no-hair" theorem. Solving Einstein's equations exactly for the gravitational radiation produced would be prohibitively expensive computationally. Instead, we rely on a series of analytic approximations: geodesic motion for the $\mathrm{CO}$ in the extreme-massratio limit, GW propagation unaffected by the boson-star spacetime, and direct identification of Schwarzschild coordinates with flat-space spherical coordinates. These approximations, the "particle on a string" approach, allow a direct application of multipole-moment expansions for both the energy and angular-momentum losses and the waveform itself. We then drop all but the mass-quadrupole moment terms in these expansions. These approximations cannot be fully justified theoretically in the highly relativistic, strong-field regime, but direct comparison with rig- orous TSN calculations in the black-hole case shows much better agreement than one might have expected $[23,24]$. We then assume that the energy and angular momentum are changing adiabatically on time scales much longer than an orbital period. This approximation, valid in the extrememass-ratio limit except during the plunge itself, allows us to apply a hybrid two-stage approach. In the first stage, differential equations for orbital elements $p$ and $e$ are obtained by relating these quantities to $E$ and $L$ with exact analytic derivatives. These equations are then integrated on a radiation-reaction time scale, with a time step that is correspondingly long compared to an orbital period. In the second stage, the orbit of the CO, $r(t)$ and $\phi(t)$, is computed with small time steps by solving the geodesic equations and linearly interpolating the trajectories $\{p(t), e(t)\}$ produced in the first stage. The hybrid approach allows us to calculate real-space trajectories and waveforms much more quickly than would be possible using the same short time step for both stages.

As anticipated, the waveforms produced by this method exhibit distinctive features that allow them to be readily distinguished from those produced during EMRIs into black holes. In the model considered here, the boson-star inspiral is identical to a black-hole inspiral until the $\mathrm{CO}$ falls over the angular-momentum barrier. If LISA observed GWs from part of an inspiral including this plunge, the parameters of the exterior black-hole spacetime could be determined very accurately using black-hole EMRI templates from the part of the inspiral up until plunge. The "smoking gun" for a boson-star inspiral would be that GWs from the inspiral persist after the plunge. This persistence could be seen using, for instance, a time-frequency analysis of the LISA data stream. GWs from an event like this could not be mistaken for an inspiral into a black hole with different parameters, because the early stages of the inspiral are identical to the black-hole inspiral. If only the post-plunge stage of a boson-star inspiral were seen, it is not clear whether this could be mistaken for a black-hole EMRI without a proper Fisher-matrix analysis.

The waveforms produced in this paper are highly approximate, far too crude to use in any attempts at matched filtering for LISA. Nonetheless, they may serve as substitutes for purposes of scoping out the data analysis and design specification until better waveforms are available. Improved waveforms might result from refining our method, for example, by incorporating higher-order multipole moments in our expansions. Press developed an improved formula for the GW field $h_{i j}^{\mathrm{TT}}$ that accounts for time delays in the source, as well as some of the relativistic effects provided by the higher moments [32]. Efforts are underway to adapt this formula to the "particle on a string" approach [25,33]. Analysis of higher harmonics of the waveform might also prove to be a useful way of distinguishing EMRIs into black holes and boson stars. These higher harmonics become comparable to the fundamental 
$m=2$ mode at high eccentricities [26]. One might expect the sudden increase in eccentricity at plunge to manifest itself in frequency space as a sharp rise in the higher harmonics. Only a rigorous comparison of our waveforms with the LISA noise curve can truly determine whether LISA can differentiate between black-hole and boson-star EMRIs. We hope to conduct such a comparison in the near future using Synthetic LISA, an actual simulation of the LISA interferometer that models its response to incident gravitational waves [34].

Going beyond these approximations to produce waveforms suitable for matched filtering by LISA will involve the use of perturbation theory in the TSN formalism. The resulting partial differential equations for a generic boson star should be separable in the spherical case, and we plan to consider this problem in the near future. Hopefully, these improvements or others will provide the tools to calculate accurate waveform templates in time for LISA's great attempt at "holiodesy," the mapping of spacetimes about the compact objects in galactic centers. Including exotic waveforms such as those produced by boson stars in our suite of templates will ensure that LISA will not miss the opportunity to discover something truly fundamental.

\section{ACKNOWLEDGMENTS}

We wish to thank L. Lindblom and E. S. Phinney for useful conversations. Micah Solomon also provided help in preparing the appendix. Kesden was supported by the NASA Graduate Student Research Program. J. G.'s work was supported by NASA Grants No. NAG5-12384 and No. NAG5-10707. This work was supported in part by DoE No. DE-FG03-92-ER40701 and NASA No. NAG59821.

\section{APPENDIX: ERROR ASSOCIATED WITH THE QUADRUPOLE APPROXIMATION}

A key approximation made in Sec. IV was to drop all but the mass-quadrupole terms from the multipole-moment expansions of the GW field, energy flux, and momentum flux in Eqs. (17)-(19). For circular orbits in the Schwarzschild metric, these expansions can be expressed
TABLE I. Coefficients describing the contribution of higherorder multipole moments to the energy flux of the inspiraling $\mathrm{CO}$.

\begin{tabular}{lcc}
\hline \hline$l$ & $A_{l}$ & $B_{l}$ \\
\hline 2 & 6.400 & 1.067 \\
3 & 6.458 & 4.571 \\
4 & 14.966 & 1.250 \\
\hline \hline
\end{tabular}

as power series in $M / r$ with coefficients that can be calculated analytically. To illustrate, the contribution of higherorder mass and current multipole moments to the energy flux is given by

$$
\frac{d E}{d(t / M)}= \begin{cases}A_{l} \eta(r / M)^{-(l+3)}, & \text { mass multipoles, } \\ B_{l} \eta(r / M)^{-(l+4)}, & \text { current multipoles, }\end{cases}
$$

where the dimensionless coefficients $A_{l}$ and $B_{l}$ [35] are listed in Table I. Neglecting the higher-order terms is clearly justified at large radii, but the expansion parameter $M / r=1 / 6$ is not particularly small at the ISO. It is still small enough, however, so that the next largest terms which happen to be the $l=3$ and $l=4$ mass multipole moments provide only $16.8 \%$ and $6.5 \%$ corrections, respectively. We continue to drop the higher-order terms as the $\mathrm{CO}$ plunges past the ISO into the interior of the boson star. If the singular behavior of Eq. (A1) held within the boson star the expansion would become formally divergent at $r=M$, but fortunately this is not the case. Outside the boson star the angular frequency $\Omega$ is Keplerian, $M \Omega=(r / M)^{-3 / 2}$, but in the interior $\Omega$ is a monotonically increasing function of $r / M$. As such, the higher-order terms in the multipolemoment expansions which involve increasingly more time derivatives become increasingly steep functions of $r / M$. The multipole-moment expansion is therefore perfectly regular at the origin, and the quadrupole approximation again becomes more accurate at small radii. The errors associated with this approximation, while excessive for the purposes of data analysis, are acceptable for an initial search for qualitative features of a boson-star EMRI.
[1] D. F. Torres, S. Capozziello, and G. Lambiase, Phys. Rev. D 62, 104012 (2000).

[2] Y. Yuan, R. Narayan, and M. J. Rees, Astrophys. J. 606, 1112 (2004).

[3] F.E. Schunck and A. R. Liddle, Phys. Lett. B 404, 25 (1997).

[4] H. Falcke, F. Melia, and E. Agol, Astrophys. J. 528, 13 (2000).

[5] D. Richstone et al., Nature (London) 395, A14 (1998).
[6] J. Kormendy and K. Gebhardt, in Proceedings of the 20th Texas Symposium on Relativistic Astrophysics, edited by H. Martel and J.C. Wheeler, AIP Conf. Proc. No. 586 (AIP, New York, 2002).

[7] E. Maoz, Astrophys. J. Lett. 447, L91 (1995).

[8] N. Weinberg, M. Milosavljevic, and A. Ghez, astro-ph/ 0404407.

[9] S. Sigurdsson and M. J. Rees, Mon. Not. R. Astron. Soc. 284, 318 (1997). 
[10] S. Sigurdsson, Classical Quantum Gravity 14, 1425 (1997).

[11] T. D. Lee and Y. Pang, Phys. Rep. 221, 251 (1992).

[12] R. Friedberg, T. D. Lee, and Y. Pang, Phys. Rev. D 35, 3640 (1987).

[13] F. D. Ryan, Phys. Rev. D 55, 6081 (1997).

[14] M. Colpi, S. L. Shapiro, and I. Wasserman, Phys. Rev. Lett. 57, 2485 (1986).

[15] R. O. Hansen, J. Math. Phys. (N.Y.) 15, 46 (1974).

[16] F. D. Ryan, Phys. Rev. D 52, 5707 (1995).

[17] R. Friedberg, T. D. Lee, and Y. Pang, Phys. Rev. D 35, 3658 (1987).

[18] R. M. Wald, General Relativity (University of Chicago Press, Chicago, 1984).

[19] S. Chandrasekhar, The Mathematical Theory of Black Holes (Oxford University Press, New York, 1992).

[20] K. S. Thorne, Rev. Mod. Phys. 52, 299 (1980).

[21] C. W. Misner, K. S. Thorne, and J. A. Wheeler, Gravitation (W. H. Freeman and Company, New York, 1973).

[22] L. S. Finn and K. S. Thorne, Phys. Rev. D 62, 124021
(2000).

[23] K. Glampedakis, S. A. Hughes, and D. Kennefick, Phys. Rev. D 66, 064005 (2002).

[24] S. Babak, H. Fang, J. R. Gair, and K. Glampedakis (to be published).

[25] T. Creighton, J. R. Gair, S. A. Hughes, and M. Vallisneri (to be published).

[26] L. Barack and C. Cutler, Phys. Rev. D 69, 082005 (2004).

[27] J. R. Gair and K. Glampedakis (to be published).

[28] S. A. Teukolsky, Astrophys. J. 185, 635 (1973).

[29] M. Sasaki and T. Nakamura, Prog. Theor. Phys. 67, 1788 (1982).

[30] C.D. Murray and S.F. McDermott, Solar System Dynamics (Cambridge University Press, New York, 1999).

[31] K. Glampedakis and D. Kennefick, Phys. Rev. D 66, 044002 (2002).

[32] W. H. Press, Phys. Rev. D 15, 965 (1977).

[33] J. R. Gair and H. Fang (unpublished).

[34] M. Vallisneri, Phys. Rev. D 71, 022001 (2005).

[35] M. Kesden and M. Solomon (to be published). 